\title{
Estimated dietary vitamin D intake during pregnancy
}

\author{
R.M. Alhomaid, M.S. Mulhern, L. Cassidy, B. Mullan, A. McGuckin and M.T. McCann \\ Northern Ireland Centre for Food and Health (NICHE), Ulster University, Coleraine, BT52 1SA, UK
}

Vitamin D is involved in calcium and phosphate homeostasis and is essential for the maintenance of bone health ${ }^{(1)}$. Insufficient vitamin D intake has significant consequences for maternal and neonatal health. In Europe, maternal vitamin D intake has been reported to fall below the recommendations ${ }^{(2-3)}$. Higher BMI is associated with lower status of vitamin D but it is unclear if dietary intakes vary according to BMI, particularly during pregnancy. Some studies have reported higher intakes in obese pregnant women relative to normal weight women ${ }^{(4)}$, whilst others have reported lower vitamin D intakes in obese compared to non-obese women ${ }^{(5)}$.

The aim of this study was to assess and compare maternal dietary vitamin D intake among normal weight, overweight and obese pregnant women.

Data collected from an ongoing double-blinded randomised vitamin D intervention study (MO-VITD) were used for analysis. Pregnant women without pregnancy complications, aged $>18$ years and having a singleton pregnancy were recruited between January 2016 and December 2016. All participants completed a validated vitamin D food frequency questionnaire (FFQ) ${ }^{(6)}$ at approximately 28 weeks gestation. Data from 80 pregnant women (43 normal weight, 20 overweight, 17 obese) were included in the current analysis.

The mean daily intake of vitamin D from food sources during pregnancy was $4.91 \mu \mathrm{g} / \mathrm{d}$. Obese pregnant women had a significantly lower dietary vitamin $\mathrm{D}$ intake compared to normal weight women $(3 \cdot 19 \mathrm{vs} .5 .57 \mu \mathrm{g} / \mathrm{day} ; \mathrm{P}=0 \cdot 037)$. There was a significant negative correlation between maternal BMI and dietary vitamin $\mathrm{D}$ intake $(\mathrm{r}=-0 \cdot 202 ; \mathrm{P}=0 \cdot 036)$. When analysed at food level, reported vitamin D intake from fish, cereal, eggs and butter was $1.48,1.33,0.97$ and $0.37 \mu \mathrm{g} / \mathrm{d}$ respectively. Breakfast cereals were the greatest contributor to vitamin D intake $(27 \%)$ and only within the 'Fish' consumption group was there a significant difference in intakes across BMI categories, with obese pregnant women having a lower fish intake compared with normal weight women (1.95 vs. $0.66 \mu \mathrm{g} / \mathrm{day} ; \mathrm{P}=0.010)$.

The findings of this study are in agreement with other European research and demonstrate that maternal dietary vitamin D intakes are low. Maternal obesity is shown to be associated with dietary vitamin D intake. These findings support the guidelines for vitamin D supplementation during pregnancy.

1. Civitelli R, Ziambaras K (2011) J Endocrinol Invest 34(7), 3-7.

2. Brembeck P, Winkvist A, Olausson H (2013) Br J Nutr 110(5), 856-864.

3. Haggarty P, Campbell DM, Knox S et al., (2013) Br J Nutr 109(5), 898-905.

4. Karlsson T, Andersson L, Hussain A et al. (2015) Clin Nutr 34(5), 892-898.

5. Scholl TO, Chen X (2009) Early Hum Dev 85(4), 231-234.

6. Weir RR, Carson EL, Mulhern MS et al. (2016) J Hum Nutr Diet 29(2), 255-261. 